\title{
ULTRAVIOLET OBSERVATIONS OF RAPIDLY ROTATING B-TYPE STARS
}

\author{
SARA R. HEAP \\ Goddard Space Flight Center, Greenbelt, Md., U.S.A.
}

\begin{abstract}
Evidence of anomalous $\mathrm{C}-\mathrm{N}$ abundances in the atmospheres of six rapidly rotating B-type stars has been found from an LTE analysis of ultraviolet line spectra obtained by Copernicus. Such anomalies are in the direction (carbon depletion, nitrogen enhancement) predicted by Paczyński (1971), but the extent of the anomaly grows toward earlier spectral type, contrary to Paczyński's predictions.
\end{abstract}

\section{DISCUSSION}

Garrison: I would like to comment that $\theta$ Car is very obviously very peculiar in the visual region as well. It obviously is a very nitrogen rich star. There are some O-type stars which are nitrogen rich and carbon poor. However, none of them are emission-line stars, as far as I know, and not all are rapid rotators. Perhaps the effect you are seeing here is somehow related to those stars and is not a rotation effect.

Heap: Yes, I agree.

Conti: Interestingly enough, there is a small group of main sequence O-type stars that also have a property of enhanced nitrogen and deficient carbon. However, none are emission-line stars, nor are they among the most rapid rotators. The origin of the anomaly is not certain.

Henize: My spectra in the ultraviolet for $\theta$ Car show that the C IV line at $\lambda 1548$ is very weak for a B0 star. Another B0 star with quite weak CIV is HR 1887, but I know nothing about its rotational characteristics. It is not a $\mathrm{Be}$ star.

Peters: HR 1887 forms a visual binary with HR 1886. I have analyzed HR 1887 using the Princeton model atmospheres and find that the carbon and nitrogen abundances are quite comparable to those in the sun.

Snijders: The results by Kamp show that for the Si III-Si IV ionization balance which you used as an effective temperature criterion, LTE is a good approximation. However for carbon and nitrogen, the importance of non-LTE effects is unknown so far. The increase of the abundance deviations you find with increasing effective temperature resembles the behavior of the LTE abundances derived from the $\mathrm{Mg} \mathrm{II}$ 4481 line as discussed by Mihalas. Especially the different abundances found from different nitrogen ions is worrisome. Extending your work to standard slow rotators like $\gamma$ Peg and $\tau$ Sco might clarify some of these problems.

Heap: Yes, I agree.

Latham: There is another mechanism that may be able to explain CNO abundance anomalies. It requires a close birary companion which was more massive and has already evolved enough to expand and transfer CNO processed mass onto the surface of the star we see now. Can you rule out this mechanism?

Heap: As far as I know, only one of the six stars studied, $\zeta$ Cen, is a binary. Perhaps, $\lambda$ Sco is also.

Hummer: I would be worried about using strong lines for this kind of analysis because of the possibility of getting effects from atmospheric motion.

Heap: Except for the case of $\theta$ Car, the ultraviolet line spectra did not show any blue shifts or asymmetries.

Peters: How sensitive are your abundances to your adopted Stark broadening parameters?

Heap: In the calculations for the strengths of the carbon and nitrogen lines, I used Sahal-Brechot and Segre's values for the electron damping constant. Had I excluded electron damping, the derived abundances would have been raised by up to a factor of two. 